\title{
理论研究 “受阻路易斯酸碱对”催化的烯醇硅醚氢化反应机理
}

\author{
王英辉 $a$ 魏思敏 $* b$ 段金伟 $a$ 王康 ${ }^{a}$ \\ $\left({ }^{a}\right.$ 长安大学 理学院 西安 710064) \\ ( $b$ 陕西中医药大学 陕西中药资源产业化省部共建协同创新中心 咸阳 712083)
}

\begin{abstract}
摘要 “受阻路易斯酸碱对” (FLPs)催化的烯醇硅醚氢化反应是一类重要的直接合成醇类化合物的方法, 然而目前其 反应机理仍不明确. 基于此，以乙基取代的全氟苯基硼作为路易斯酸 $\left(\mathrm{Et}-\mathrm{B}\left(\mathrm{C}_{6} \mathrm{~F}_{5}\right)_{2}\right)$, 三叔丁基膦 $(t-\mathrm{Bu} 3 \mathrm{P})$ 作为路易斯碱, 烯醇硅醚化的苯乙酮(Me-TMS)作为底物建立了模型反应, 并使用密度泛函理论系统研究了其催化氢化反应机理. 结果 显示: FLPs 催化的烯醇硅醚氢化反应从 Et-B $\left(\mathrm{C}_{6} \mathrm{~F}_{5}\right)_{2}$ 和 $t$-Bu3 $\mathrm{P}$ 形成 B-P-FLPs 开始, 随后会依次经过 $\mathrm{H}_{2}$ 裂解, $\mathrm{H}^{+}$和 $\mathrm{H}^{-}$转 移等过程, 其中 $\mathrm{H}^{+}$转移为决速步, $\mathrm{H}^{-}$转移无势垒, B-P-FLPs 生成及 $\mathrm{H}^{+}$转移是吸热反应. 升高温度不利于氢化反应发生, 但是增大压力可促进反应进行. 底物取代基效应会影响 $\mathrm{H}^{-}$转移过程, 可能使反应不发生.
\end{abstract}

关键词＼cjkstart受阻路易斯酸碱对；烯醇硅梄；氢化反应；密度泛函理论

Mechanism of Silyl Enol Ethers Hydrogenation Catalysed by Frustrated Lewis Pairs: A Theoretical Study

\author{
Yinghui Wang $^{a} \quad$ Simin Wei*, $^{*} \quad$ Jinwei Duan $^{a} \quad$ Kang Wang $^{a}$ \\ $\left({ }^{a}\right.$ College of Science, Chang'an University, Xi'an 710064, China) \\ $\left({ }^{b}\right.$ Co-construction Collaborative Innovation Center for Chinese Medicine Resources Industrialization by \\ Shaanxi \& Education Ministry, Shaanxi University of Chinese Medicine, Xianyang 712083, China)
}

\begin{abstract}
Silyl enol ethers have attracted enormous attention as they could serve as a test bed for the development of novel frustrated Lewis pairs (FLPs) catalytic systems. However, the reaction mechanism of hydrogenation catalysed by metal-free FLPs for these compounds to the corresponding secondary alcohols remains elusive to a large extent in previous studies. We thus performed a thorough investigation on the reaction mechanism by density functional theory (DFT). To illustrate the reaction mechanism of FLPs-catalysed hydrogenation for silyl enol ethers, trimethyl((1-phenylvinyl)oxy)silane (Me-TMS) was chosen as the prototype substrate and toluene as the solvent, where the FLPs were generated by ethylbis(perfluorophenyl)borane $\left(\mathrm{Et}-\mathrm{B}\left(\mathrm{C}_{6} \mathrm{~F}_{5}\right)_{2}\right)$ and tri-tert-butylphosphine $(t-\mathrm{Bu} 3 \mathrm{P})$. The M06-2X functional in connection with $6-31+\mathrm{G}(\mathrm{d})$ basis set was used to optimize the structures of related species including in the Gibbs free energy profiles, and the energies were obtained at M06-2X/6-311++G(d,p) level of theory, where the solvent effect was simulated with the integral equation formalism, polarized continuum mode (IEF-PCM) in both calculations. Our results suggest that the FLPs-catalysed hydrogenation of silyl enol ethers in toluene begins with the formation of B-P-FLPs followed by hydrogen activation, proton transfer and hydride transfer to complete the process. It is obvious from the Gibbs free energy profile that the proton transfer is rate-determining step, the formation of B-P-FLPs and proton transfer are endothermal and the hydride transfer is no barrier. This indicates that the amount of $\mathrm{H}_{2}$ and prototype substrate have significant influence on the FLPs-catalysed hydrogenation of silyl enol ethers. A higher temperature $(328.15 \mathrm{~K})$ is disadvantageous to hydrogenation reaction catalysed by FLPs but the reaction could be accelerated under higher pressure $(4040 \mathrm{kPa})$. The Gibbs free energy profile calculations for trimethyl((1-phenylprop-1-en-1-yl)oxy)silane (Et-TMS) and tert-butyldimethyl((1-phenylvinyl)oxy)silane (Me-TBS) reveal that substituent group may inhibit the hydride transfer as the absence of a suitable construction for $\mathrm{R}-\mathrm{H}^{-}$-transfer, where the hydride does not direct to the $\mathrm{C}^{+}$of silyl enol ethers and the distance between $\mathrm{C}^{+}$and hydride is longer. These results would be helpful to design another novel FLPs-catalysed hydrogenation reaction for silyl enol ethers.
\end{abstract}

Keywords frustrated Lewis pairs; silyl enol ethers; hydrogenation; density functional theory

\footnotetext{
*E-mail: weisimin@iccas.ac.cn

Received May 28, 2021; published August 10, 2021.

Supporting information for this article is available free of charge via the Internet at http://sioc-journal.cn.

Project supported by the National Natural Science Foundation of China (Nos. 21705029, 21804066), the Young Talent Fund of University Association for Science and Technology in Shaanxi, China (No. 20190307), the Natural Science Foundation of Shaanxi Province (No. 2021JQ-221), the Fundamental Research Funds for the Central Universities, CHD (No. 300102120303) and the Special Scientific Research Plan of Education Department of Shaanxi Province (No. 19JK0233).

项目受国家自然科学基金(Nos. 21705029, 21804066)、陕西省高校科协青年人才托举计划(No. 20190307)、陕西省自然科学基金(No. 2021JQ-221)、长 安大学中央高校基本科研业务费专项资金(No. 300102120303)和陕西省教育厅自然科学研究项目(No. 19JK0233)资助.
} 


\section{1 引言}

不饱和有机化合物的催化加氢反应是化学中应用 最广泛的反应之一 ${ }^{[1-8]}$. 长期以来, 各国的研究者致力于 开发不同类型的催化氢化反应体系, 过渡金属催化剂在 该领域一直占主导地位, 并已经取得了很多辉煌的成 就 ${ }^{[9-17]}$. 直到 2006 年, Stephan 小组 ${ }^{[18]}$ 发现 “受阻路易斯 酸碱对”(frustrated Lewis pairs, 简称为 FLPs) 在 298.15 K 时可以使氢气发生异裂, 其还原形式在 $373.15 \mathrm{~K}$ 时可释 放氢气, 首次实现了非金属催化剂对氢气的可逆活化. FLPs 的出现为催化氢化反应提供了一种全新的策略. 因为此方法不需要使用钓、铑、铱、钯等贵金属, 可以 有效避免金属残留和催化剂失活等问题, 目前已引起各 国科研工作者极大的研究兴趣 ${ }^{[19]}$. 截止目前, FLPs 已作 为催化剂被应用于亚胺、烯胺、烯烃、多芳香烃、炔烃、 酮和醛等不饱和化合物的氢化反应 ${ }^{[12,20-30]}$; 此外, 有报 道显示: FLPs 还能活化 $\mathrm{N}_{2} 、 \mathrm{CO}_{2} 、 \mathrm{SO}_{2} 、 \mathrm{NO} 、 \mathrm{CO} 、 \mathrm{~N}_{2} \mathrm{O}$ 等小分子[31-35], 进一步扩展了 FLPs 化学的范畴.

烯醇硅醚常被用作开发新的 FLPs 催化氢化反应体 系. 与亚胺和 $N$-杂环相比, 虽然烯醇硅醚可以在 FLPs 中充当 Lewis 碱的角色, 但是由于烯醇硅醚的碱度较低, 常常需要加入额外的路易斯碱用于氢气活化. 比如: 2008 年, Erker 小组 ${ }^{[3]}$ 使用 1,8-双 (二苯基膦基)菜和全氟 苯基硼 $\left(\mathrm{B}\left(\mathrm{C}_{6} \mathrm{~F}_{5}\right)_{3}\right)$ 形成的 FLPs 首次实现了烯醇硅醚的催 化氢化反应; 2012 年, Paradies 和同事 ${ }^{[37]}$ 使用[2.2]-对环 己烷衍生的二膦作为 FLPs 中路易斯碱部分与 $\mathrm{B}\left(\mathrm{C}_{6} \mathrm{~F}_{5}\right)_{3}$ 形成 FLPs 氢化了烯醇硅醚; 随后我们课题组 ${ }^{[26,38]}$ 通过 原位生成的轴向手性硼烷作为 Lewis 酸, $t$ - $\mathrm{Bu}_{3} \mathrm{P}$ 作为 Lewis 碱形成 FLPs 实现了烯醇硅醚手性加氢反应(图 1). 然而, 相对于快速发展的 FLPs 催化烯醇硅醚氢化反应, 这类反应的机理研究相对滞后, 据我们所知, 目前 FLPs 催化的烯醇硅醚氢化反应机理还未见报道, 这在一定程 度上限制了该类型反应的发展。

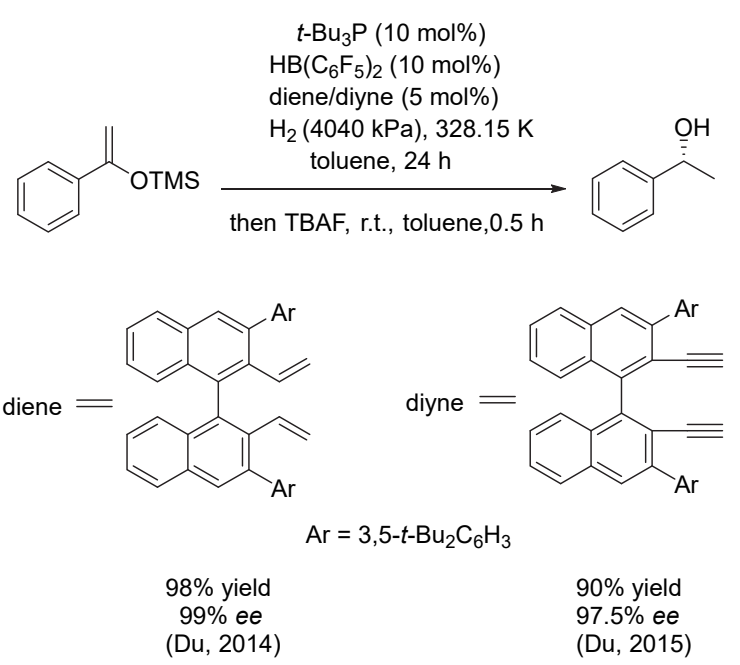

图 1 文献报道的 “受阻路易斯酸碱对”催化的烯醇硅醚氢化反应 Figure 1 FLPs-catalyzed hydrogenation of silyl enol ethers developed by previous study
基于以上背景, 我们以乙基取代的全氟苯基硼作为 路易斯酸 $\left(\mathrm{Et}-\mathrm{B}\left(\mathrm{C}_{6} \mathrm{~F}_{5}\right)_{2}\right)$, 三叔丁基膦 $\left(t-\mathrm{Bu}_{3} \mathrm{P}\right)$ 作为路易斯 碱, 烯醇硅醚化的苯乙䣳(Me-TMS)作为底物建立了模 型反应, 并使用密度泛函理论(DFT), 在 IEF-PCM M06-2X/6-31+G(d)理论水平上对 FLPs 催化的烯醇硅醚 氢化反应中各反应物、中间体、过渡态和产物的构型进 行优化; 温度效应、压力效应以及底物取代基效应对氢 化反应吉布斯自由能的影响也被系统评价. 我们的这些 研究结果将会为后期设计更加高效的 FLPs 催化烯醇硅 醚氢化反应提供理论指导.

\section{2 结果与讨论}

\section{1 模型反应与化学计量比}

根据我们前期的结果 ${ }^{[26,38]}$ : 在 $328.15 \mathrm{~K}$, 压力为 $4040 \mathrm{kPa}$, 反应溶剂为甲苯时, 手性二烯/炔配体与全氟 苯基硼氢 $\left(\mathrm{HB}\left(\mathrm{C}_{6} \mathrm{~F}_{5}\right)_{2}\right)$ 混合后会发生硼氢化反应原位生 成手性硼烷，手性硼烷与三叔丁基膦 $\left(t-\mathrm{Bu}_{3} \mathrm{P}\right)$ 会形成 FLPs, 并作为催化剂活化氢气使烯醇硅醚发生氢化反 应，当氢化反应完成后使用四丁基氟化铵(TBAF)脱保 护即可得到相应的醇类化合物. 本工作中, 我们拟选取 该反应体系为反应模型体系. 我们首先对模型反应体系 进行了优化, 因为手性二烯/炔配体与 $\mathrm{HB}\left(\mathrm{C}_{6} \mathrm{~F}_{5}\right)_{2}$ 的硼氢 化反应很容易发生, 所以我们直接选取两者的加和物作 为路易斯酸, 为了提高计算效率, 我们将路易斯酸简化 为乙基取代的全氟苯基硼(Et-B $\left.\left(\mathrm{C}_{6} \mathrm{~F}_{5}\right)_{2}\right)$; 三叔丁基膦作 为路易斯碱, 与 $\mathrm{Et}-\mathrm{B}\left(\mathrm{C}_{6} \mathrm{~F}_{5}\right)_{2}$ 形成 FLPs, 定义为 B-P-FLPs; 三甲基硅烷化的苯乙酮烯醇硅醚(Me-TMS) 作为反应底物, 甲苯作为溶剂进行了后续的计算(图 2). 我们优化得到的 Et- $\mathrm{B}\left(\mathrm{C}_{6} \mathrm{~F}_{5}\right)_{2}, t-\mathrm{Bu}_{3} \mathrm{P}$ 和 Me-TMS 结构如 图 3 所示. 此外, 因为 TBAF 脱保护生成醇的过程很容 易发生，所以我们未对此过程进行讨论.

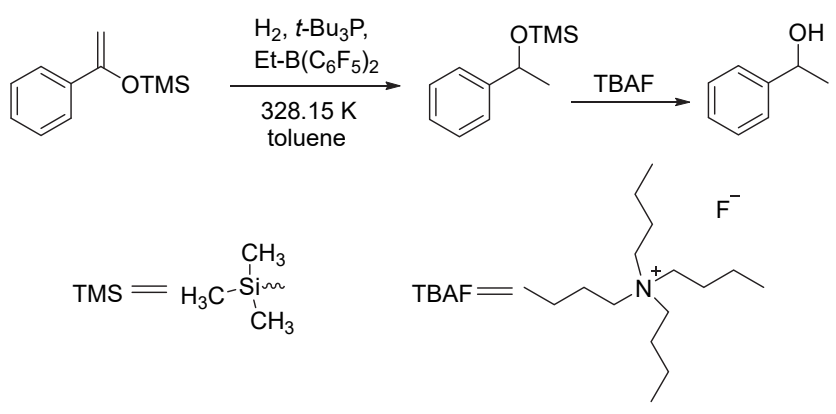

图 2 “受阻路易斯酸碱对”催化的烯醇硅梄氢化模型反应

Figure 2 Model reaction for the silyl enol ethers hydrogenation catalysed by frustrated Lewis pairs

\section{2 “硼-膦受阻路易斯酸碱对” 的形成及其催化的氢 气裂解机理}

M06-2X 泛函 ${ }^{[39]}$ 已被证明在处理有弱相互作用的体 系时能取得较好的结果 ${ }^{[40-43]}$, 并且该方法已被用于建立 


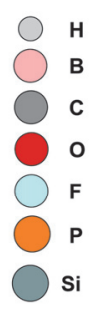

0.074

00

.



$t$-Bu3 $\mathrm{P}$

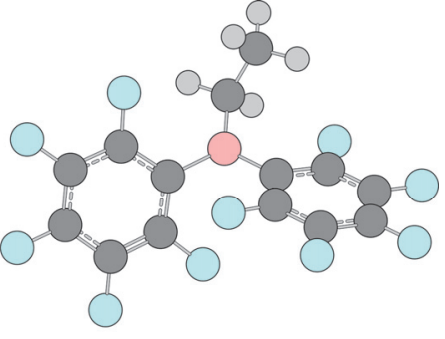

$\mathrm{Et}-\mathrm{B}\left(\mathrm{C}_{6} \mathrm{~F}_{5}\right)_{2}$

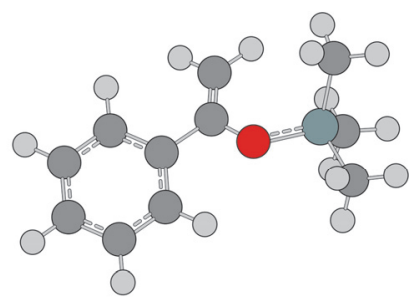

Me-TMS
图 3 在 IEF-PCM M06-2X/6-31+G(d)理论水平上优化得到的 $\mathrm{H}_{2}$ 、 Et-B $\left(\mathrm{C}_{6} \mathrm{~F}_{5}\right)_{2} 、 t-\mathrm{Bu}_{3} \mathrm{P}$ 和 Me-TMS 结构, 键长单位为 $\mathrm{nm}$

Figure 3 Optimized structures at IEF-PCM M06-2X/6-31+G(d) level of theory for $\mathrm{H}_{2}, \mathrm{Et}-\mathrm{B}\left(\mathrm{C}_{6} \mathrm{~F}_{5}\right)_{2}, t-\mathrm{Bu}_{3} \mathrm{P}$ and Me-TMS. Selected bond distances are given in $\mathrm{nm}$

FLPs 催化的氢化反应体系势能面 ${ }^{[44-49]}$, 从结果看该理 论水平下揭示的反应机理可以合理解释相关实验事实. 所以为了揭示 FLPs 催化的烯醇硅醚氢化反应机理我们 选用密度泛函 M06-2X 方法并结合 6-31+G(d)基组对其 吉布斯自由能曲线进行了理论预测. 为了得到更精确的 能量, 我们在 M06-2X/6-311++ G(d,p)理论水平下对吉 布斯自由能面中包含的各化合物进行了单点能校正. 在 优化构型以及单点能计算过程中我们充分考虑了甲苯 的溶剂化效应, 并使用积分方程形式的极化连续介质模 型(IEF-PCM) $)^{[50]}$ 进行模拟.

“受阻路易斯酸碱对” 催化不饱和化合物发生氢化 反应一般是从 FLPs 裂解氢气开始, 所以我们首先计算 了 Et-B $\left(\mathrm{C}_{6} \mathrm{~F}_{5}\right)_{2}$ 与 $t-\mathrm{Bu}_{3} \mathrm{P}$ 形成 FLPs 及其裂解氢气过程的 吉布斯自由能曲线. 根据文献报道 ${ }^{[49,51]}$ : 如果反应底物 中包含有氧 $(\mathrm{O})$ 、氮 $(\mathrm{N})$ 等原子时, 这些原子可以充当路 易斯碱的角色与全氟苯基硼形成 “受阻路易斯酸碱对”。 因为我们的模型底物 Me-TMS 中包含有氧原子, 所以我 们首先探讨了 Et-B $\left(\mathrm{C}_{6} \mathrm{~F}_{5}\right)_{2}$ 与其形成 FLPs 催化烯醇硅醚 氢化反应的可能性. 根据我们前期的实验研究 ${ }^{[26]}$ : 当在 体系中只加入全氟苯基氢 $\left(\mathrm{HB}\left(\mathrm{C}_{6} \mathrm{~F}_{5}\right)_{2}\right)$ 和双烯配体形成 手性硼时并不能催化该氢化反应, 然而当加入烷基取代 的膦化合物时该氢化反应可顺利进行. 因此可以确认: 在该反应体系中可能并不生成硣-氧受阻路易斯酸碱对 (B-O-FLPs), 即使形成 B-O-FLPs 也不能催化烯醇硅醚 发生氢化反应. 此外, 有研究显示 ${ }^{[51]}$ : 包含有氧原子的 溶剂分子, 如乙醚等也可以作为路易斯碱与全氟苯基硼
形成 FLPs, 本工作中我们考察的烯醇硅醚氢化反应是 在甲苯中进行, 因此并不存在 $\mathrm{Et}-\mathrm{B}\left(\mathrm{C}_{6} \mathrm{~F}_{5}\right)_{2}$ 与溶剂分子 形成 FLPs 催化裂解氢气的可能. 所以, 最终在计算中 我们只考虑 $\mathrm{Et}-\mathrm{B}\left(\mathrm{C}_{6} \mathrm{~F}_{5}\right)_{2}$ 与 $t-\mathrm{Bu}_{3} \mathrm{P}$ 形成的 FLPs (B-PFLPs)活化并裂解氢气的路径.

首先, 我们在 $298.15 \mathrm{~K}, 101 \mathrm{kPa}$ 时计算了 $\mathrm{Et}-\mathrm{B}\left(\mathrm{C}_{6} \mathrm{~F}_{5}\right)_{2}$ 与 $t-\mathrm{Bu}_{3} \mathrm{P}$ 形成 FLPs 及其裂解氢气过程的吉 布斯自由能曲线, 结果如图 4 所示(黑色). 图中以 $\mathrm{Et}-\mathrm{B}\left(\mathrm{C}_{6} \mathrm{~F}_{5}\right)_{2} 、 t-\mathrm{Bu}_{3} \mathrm{P} 、 \mathrm{H}_{2}$ 和反应底物的能量之和为能量 零点. 从图中可以看出: $\mathrm{Et}-\mathrm{B}\left(\mathrm{C}_{6} \mathrm{~F}_{5}\right)_{2}$ 与 $t-\mathrm{Bu}_{3} \mathrm{P}$ 会首先形 成 “受阻路易斯酸碱对” B-P-FLPs, 当在体系中加入 $\mathrm{H}_{2}$ 后, $\mathrm{H}_{2}$ 分子会和 B-P-FLPs 中的 B 中心通过配位相互作 用形成 $\mathrm{R}-\mathrm{H}_{2}$-split，然后 $\mathrm{R}-\mathrm{H}_{2}$-split 会通过过渡态 TS- $\mathrm{H}_{2}$-split 形成两性离子对中间体 $\mathrm{P}-\mathrm{H}_{2}$-split. 在 B-P-FLPs 中, 嗍-膦键 $(B-P)$ 键长为 $0.367 \mathrm{~nm}$, 这和文献 报道的 B-P FLPs 键长一致 ${ }^{[37]}, \mathrm{B}-\mathrm{P}$ 键键长明显比两者 形成的路易斯酸碱加合物要长; 同时 B-P-FLPs 中位于 Et-B $\left(\mathrm{C}_{6} \mathrm{~F}_{5}\right)_{2}$ 中心的 $\mathrm{BC}_{3}$ 保持近平面构型, 这表明在 B-P-FLPs 中没有 $\mathrm{B}-\mathrm{P}$ 配位键形成; $\mathrm{Et}-\mathrm{B}\left(\mathrm{C}_{6} \mathrm{~F}_{5}\right)_{2}$ 与 $t-\mathrm{Bu}_{3} \mathrm{P}$ 形成 FLPs 需要吸热 $16.5 \mathrm{~kJ} / \mathrm{mol}$. 在 R- $\mathrm{H}_{2}$-split 中氢气分 子与 $\mathrm{Et}-\mathrm{B}\left(\mathrm{C}_{6} \mathrm{~F}_{5}\right)_{2}$ 中的 $\mathrm{B}$ 原子以及 $t-\mathrm{Bu}_{3} \mathrm{P}$ 中 $\mathrm{P}$ 的距离分 别为 $0.319,0.301$ 和 $0.319 \mathrm{~nm}$; 氢气中两个氢原子之间 的距离 $(0.0742 \mathrm{~nm})$, 比自由的氢气 $(0.0737 \mathrm{~nm})$ 稍长, 这 表明在初始形成的 $\mathrm{R}-\mathrm{H}_{2}$-split 中, 氢气分子已被 B-P-FLP 活化. 在此基础上, 随后的氢气裂解过程只需 要经过 $85.7 \mathrm{~kJ} / \mathrm{mol}$ 的能垒就能发生, 整个氢气裂解过程 需吸收能量 $8.7 \mathrm{~kJ} / \mathrm{mol}$. 在过渡态 TS- $\mathrm{H}_{2}-\mathrm{split}$ 中, 氢气分 子中两个氢原子间的距离进一步增加到 $0.081 \mathrm{~nm}$ ，部分

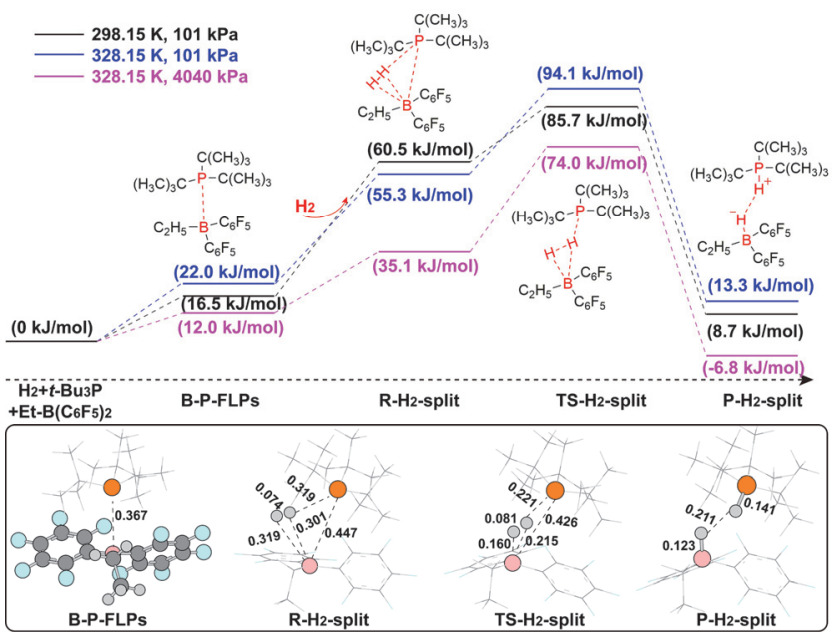

图 4 优化得到的 FLPs 形成及其催化的 $\mathrm{H}_{2}$ 裂解吉布斯自由能曲线, 其中优化的结构及其相对能量分别在 IEF-PCM M06-2X/6-31+ G(d) 和 IEF-PCM M06-2X/6-311++G(d,p) 理论水平获得, 键长单位为 $\mathrm{nm}$ Figure 4 Optimized structures as well as Gibbs free energy profile of related species involved in the generation of B-P-FLPs and $\mathrm{H}_{2}$ split. The structures and energies reported in this figure were calculated at the IEF-PCM M06-2X/6-31+ G(d) and IEF-PCM M06-2X/6-311+ + G(d,p) level of theory, respectively. Selected bond distances are given in $\mathrm{nm}$ 
的 $\mathrm{B}-\mathrm{H}$ 键 $(0.160 \mathrm{~nm})$ 和 $\mathrm{P}-\mathrm{H}$ 键 $(0.221 \mathrm{~nm})$ 已经形成. 在 形成的两性离子对中间体 $\mathrm{P}-\mathrm{H}_{2}$-split 中, $\mathrm{B}-\mathrm{H}$ 键 $(0.123$ $\mathrm{nm})$ 和 $\mathrm{P}-\mathrm{H}$ 键 $(0.141 \mathrm{~nm})$ 完全形成, 氢气被裂解后形成 的 $\mathrm{H}^{+}$和 $\mathrm{H}^{-}$之间的距离为 $0.211 \mathrm{~nm}$, 这表明在 $\mathrm{P}-\mathrm{H}_{2}-\mathrm{split}$ 中 $\mathrm{H}_{2}$ 分子已被完全裂解.

随后, 我们在 $328.15 \mathrm{~K}, 101 \mathrm{kPa}$ 时对氢气裂解过程 中各物种的吉布斯自由能进行了计算, 结果如图 4 所示 (蓝色). 从图中可以看出: 当温度升高时, $\mathrm{Et}-\mathrm{B}\left(\mathrm{C}_{6} \mathrm{~F}_{5}\right)_{2}$ 与 $t-\mathrm{Bu}_{3} \mathrm{P}$ 形成的 B-P-FLPs 更加不稳定, 其催化的氢气裂解 能垒从 $85.7 \mathrm{~kJ} / \mathrm{mol}$ 升高至 $94.1 \mathrm{~kJ} / \mathrm{mol}$, 生成的两性离子 对中间体也不如 $298.15 \mathrm{~K}, 101 \mathrm{kPa}$ 时的稳定. 升高温度 似乎不利于氢气裂解.

接着, 我们在 $328.15 \mathrm{~K}$ 时将压力升高至 $4040 \mathrm{kPa}$ 进行了计算, 结果如图 4 所示(粉色). 在该条件下, $\mathrm{Et}-\mathrm{B}\left(\mathrm{C}_{6} \mathrm{~F}_{5}\right)_{2}$ 与 $t-\mathrm{Bu}_{3} \mathrm{P}$ 生成的 B-P-FLPs 比 $298.15 \mathrm{~K}, 101$ $\mathrm{kPa}$ 时的更加稳定, 氢气裂解的能垒也更低 (74.0 $\mathrm{kJ} / \mathrm{mol})$, 整个反应从轻微的吸热变为放热 $6.8 \mathrm{~kJ} / \mathrm{mol}$. 增大压力有助于 B-P-FLPs 裂解氢气.
2.3 两性离子对中间体与烯醇硅醚化苯乙酮 -Me-TMS 的 $\mathrm{H}^{+}$和 $\mathrm{H}^{-}$转移机理

当氢气被完全裂解之后, 烯醇硅醚化的苯乙酮会通 过配位相互作用与两性离子对中间体形成 $M M-R-H^{+}$transfer, 如图 5 所示. 该化合物中 $\mathrm{P}-\mathrm{H}^{+}$键指向烯醇硅 醚中 $=\mathrm{CH}_{2}$ 基团中不饱和的碳原子, $\mathrm{P}-\mathrm{H}^{+}$键长稍微缩 短至 $0.141 \mathrm{~nm}, \mathrm{H}^{+}$与 $=\mathrm{CH}_{2}$ 基团中的不饱和碳原子距离 为 $0.285 \mathrm{~nm}$, 烯醇硅醚中 $\mathrm{C}=\mathrm{CH}_{2}$ 双键的距离为 0.134 $\mathrm{nm}$; 因为烯醇硅醚化苯乙酮的加入是一个去稳定化的 过程, 需要吸热 $41.5 \mathrm{~kJ} / \mathrm{mol}$, 所以其会通过过渡态 MM-TS- $\mathrm{H}^{+}$-transfer 快速地将 $\mathrm{P}-\mathrm{H}^{+}$键中 $\mathrm{H}^{+}$转移到烯醇 硅醚中的 $=\mathrm{CH}_{2}$ 基团上形成 $\mathrm{MM}-\mathrm{P}-\mathrm{H}^{+}$-transfer; 在 MM-TS- $\mathrm{H}^{+}$-transfer 中, $\mathrm{H}^{+}$位于 $\mathrm{P}$ 和 $=\mathrm{CH}_{2}$ 中 $\mathrm{C}$ 原子之 间, 距离分别为 0.190 和 $0.131 \mathrm{~nm}, \mathrm{C}=\mathrm{C}$ 键长增大至 $0.141 \mathrm{~nm}$. 从吉布斯自由能曲线上看 $\mathrm{H}^{+}$转移需要克服 $125.4 \mathrm{~kJ} / \mathrm{mol}$ 的能垒, 形成的 $\mathrm{MM}-\mathrm{P}-\mathrm{H}^{+}$-transfer 是一个 更不稳定的中间体 $(99.0 \mathrm{~kJ} / \mathrm{mol})$. 在 $\mathrm{MM}-\mathrm{P}-\mathrm{H}^{+}$-transfer 中因为不饱和的 $\mathrm{C}^{+}$与 $\mathrm{B}-\mathrm{H}^{-}$键存在一定角度 $\left(92.5^{\circ}\right), \mathrm{C}^{+}$

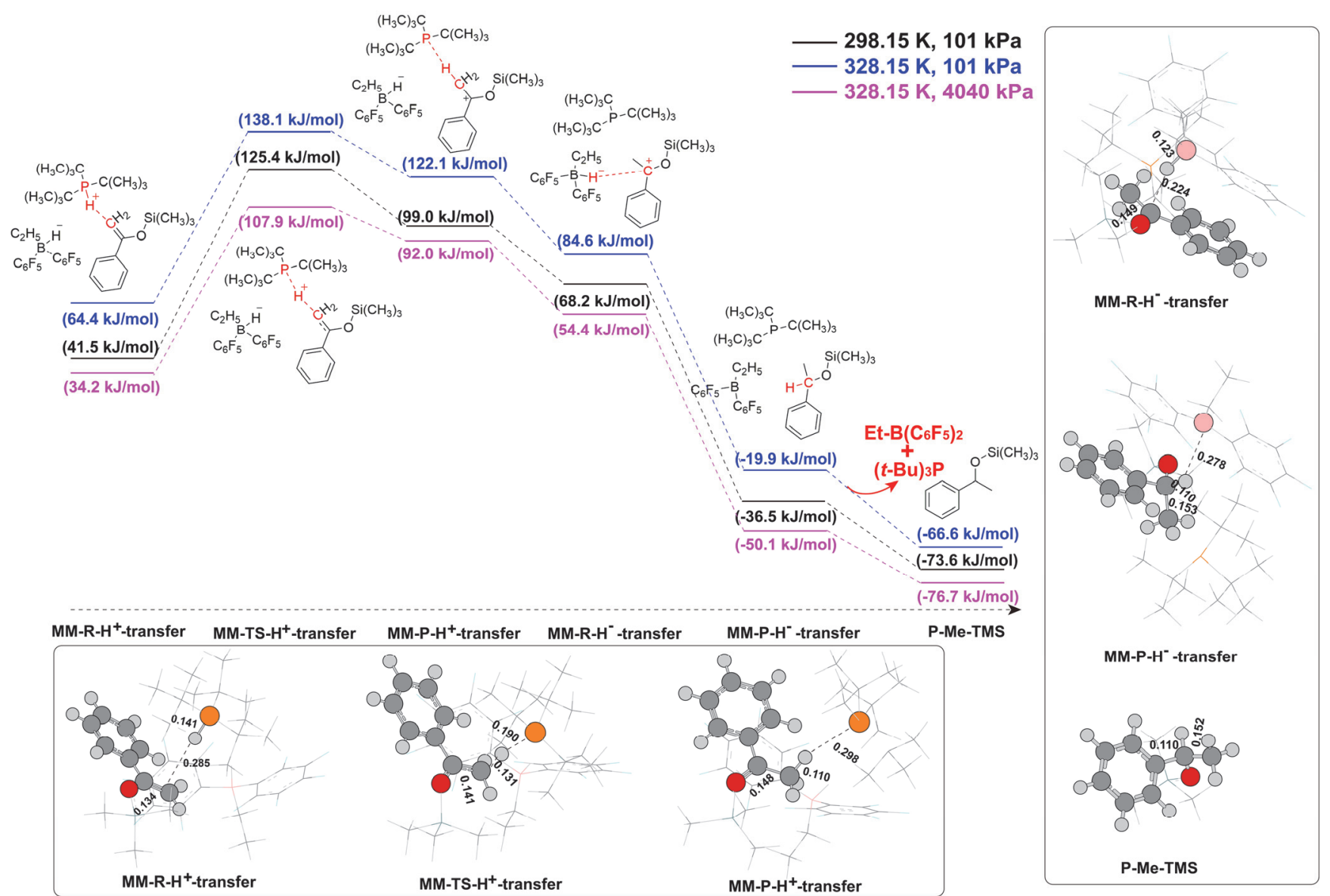

图 5 优化得到的两性离子与 Me-TMS 的 $\mathrm{H}^{+}$和 $\mathrm{H}^{-}$转移吉布斯自由能曲线, 其中优化的结构及其相对能量分别在 IEF-PCM M06-2X/6-31+ G(d) 和 IEF-PCM M06-2X/6-311 $++\mathrm{G}(\mathrm{d}, \mathrm{p})$ 理论水平获得, 键长单位为 $\mathrm{nm}$

Figure 5 Optimized structures as well as Gibbs free energy profile of $\mathrm{H}^{+}$and $\mathrm{H}^{-}$transfer between intermediate of ion pair and Me-TMS. The structures and energies reported in this figure were calculated at the IEF-PCM M06-2X/6-31+G(d) and IEF-PCM M06-2X/6-311+ + G(d,p) level of theory, respectively. Selected bond distances are given in $\mathrm{nm}$ 
与 $\mathrm{H}^{-}$的距离为 $0.413 \mathrm{~nm}, \mathrm{~B}-\mathrm{H}^{-}$为 $0.123 \mathrm{~nm}$, 该结构不 利于发生 $\mathrm{H}^{-}$转移, 所以 $\mathrm{MM}-\mathrm{P}-\mathrm{H}^{+}$-transfer 会经过重排 形成更利于发生 $\mathrm{H}^{-}$转移的中间体 MM-R- $\mathrm{H}^{-}$-transfer, 该重排过程放热 $30.8 \mathrm{~kJ} / \mathrm{mol}$. 在 $\mathrm{MM}-\mathrm{R}-\mathrm{H}^{-}$-transfer 中, B- $\mathrm{H}^{-} \cdots \mathrm{C}^{+}$角度增大至 $155.9^{\circ}$, 更趋近于直线, $\mathrm{C}^{+}$与 $\mathrm{H}^{-}$ 的距离缩短至 $0.224 \mathrm{~nm}, \mathrm{~B}-\mathrm{H}^{-}$稍微增大至 $0.123 \mathrm{~nm}$. 中 间体 MM-R-H ${ }^{-}$-transfer 并不是一个稳定的产物, 所以会 通过转移 $\mathrm{H}^{-}$使体系的能量继续下降, 势能面扫描表明 $\mathrm{H}^{-}$转移是一个无势垒的过程, 且随着 $\mathrm{H}^{-}$和 $\mathrm{C}^{+}$之间的距 离减小能量持续下降(图 6), 形成的产物 $\mathrm{MM}-\mathrm{P}-\mathrm{H}^{-}$transfer 能量为 $-36.5 \mathrm{~kJ} / \mathrm{mol}, \mathrm{H}^{-}$转移过程放热达 104.7 $\mathrm{kJ} / \mathrm{mol}$. 在 MM-P- $\mathrm{H}^{-}$-transfer 中, $\mathrm{B}-\mathrm{H}^{-}$键完全断开, 距 离为 $0.278 \mathrm{~nm}$, 与之对应的是 $\mathrm{C}^{+}$与 $\mathrm{H}^{-}$成键, 键长为 $0.110 \mathrm{~nm}$, 与普通的 $\mathrm{C}-\mathrm{H}$ 键长基本一致, 表明 $\mathrm{H}^{-}$已完 全转移至 $\mathrm{C}^{+}$上. 随后, 因为被氢化的烯醇硅醚与 $\mathrm{Et}-\mathrm{B}\left(\mathrm{C}_{6} \mathrm{~F}_{5}\right)_{2}$ 和 $t-\mathrm{Bu}_{3} \mathrm{P}$ 之间的相互作用已很弱, 所以其会 迅速解离形成最终产物 P-Me-TMS. 相对于 MM-P-H -transfer, 该解离过程还会持续放热 $(37.1 \mathrm{~kJ} / \mathrm{mol})$, 至此 体系到达能量最低点 $(-73.6 \mathrm{~kJ} / \mathrm{mol})$. 从该吉布斯自由 能曲线中我们得到的产物 P-Me-TMS 构型和我们实验 中的一致, 因此可以确认我们建立的吉布斯自由能面可 以描述 B-P-FLPs 催化的烯醇硅梄氢化反应, 也进一步 说明我们上文对于烷基取代的全氟苯基嗍结构优化的 合理性.

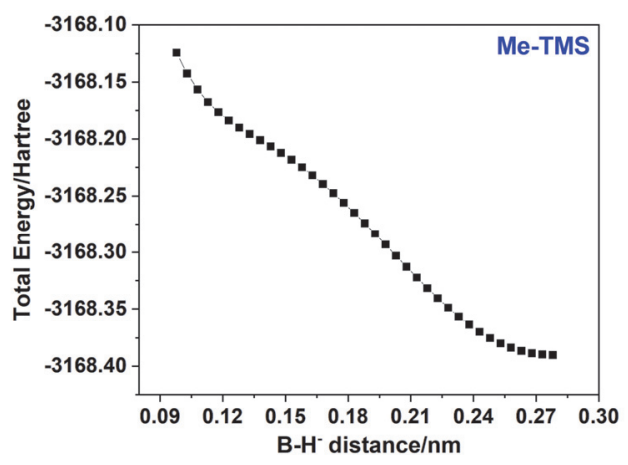

图 6 IEF-PCM M06-2X/6-31+G(d)理论水平扫描 Me-TMS 中 $\mathrm{H}^{-}$转移 势能面

Figure 6 Total energy scan of hydride transfer for Me-TMS at IEF-PCM M06-2X/6-31+G(d) level of theory

随后, 我们计算了 $328.15 \mathrm{~K}, 101 \mathrm{kPa}$ 时两性离子中 间体与烯醇硅醚发生 $\mathrm{H}^{+}$和 $\mathrm{H}^{-}$转移的吉布斯自由能曲 线. 与计算的 $\mathrm{H}_{2}$ 裂解吉布斯自由能曲线类似, 图中各物 质的能量均不同程度上升, 升高温度使 $\mathrm{H}^{+}$转移的能垒 从 125.4 上升至 $138.1 \mathrm{~kJ} / \mathrm{mol}$ (图 5). 在该温度下将压力 提高至 $4040 \mathrm{kPa}$ 发现: 吉布斯自由能曲线中各物质比 $101 \mathrm{kPa}$ 时更加稳定, $\mathrm{H}^{+}$转移的能垒降低至 $107.9 \mathrm{~kJ} / \mathrm{mol}$. 考虑到吉布斯自由能面中 $\mathrm{H}_{2}$ 裂解的能垒远小于 $\mathrm{H}^{+}$转 移, 所以 $\mathrm{H}^{+}$转移为整个过程的决速步, 增大压力使决 速步的能垒下降, 因此可以理解我们在前期实验中增大
压力后整个反应的效率会提升.

\section{4 两性离子对中间体与烯醇硅醚一Et-TMS 和 Me-TBS 的 $\mathrm{H}^{+}$和 $\mathrm{H}^{-}$转移机理}

此外, 我们注意到, 不管是在我们前期的工作还是 在 Erker 等 ${ }^{[36]}$ 和 Paradies 等 ${ }^{[37]}$ 的工作中, 对于 FLPs 催化 的苯丙酮烯醇硅醚或者更长碳链酮的氢化反应没有进 行讨论, 并且烯醇硅醚化酮时采用的都是三甲基硅烷, 所以为了更加深入地理解 B-P-FLPs 催化的烯醇硅醚氢 化反应机理，我们还对三甲基硅烷烯醇硅醚化的苯丙酮 (Et-TMS) 及叔丁基二甲基硅烷烯醇硅醚化的苯乙酮 (Me-TBS)氢化反应机理进行了计算. 优化的 Et-TMS 和 Me-TBS 结构如图 7 所示. 与 Me-TMS 相比, Et-TMS 只 是在其烷基端增加了一个甲基，而 Me-TBS 是将三甲基 硅烷中的一个甲基用叔丁基取代. 底物的变化并不会影 响 B-P-FLPs 的形成及其氢气裂解过程, 所以吉布斯自 由能面计算我们从 Et-TMS 和 Me-TBS 与两性离子对中 间体形成复合物开始.
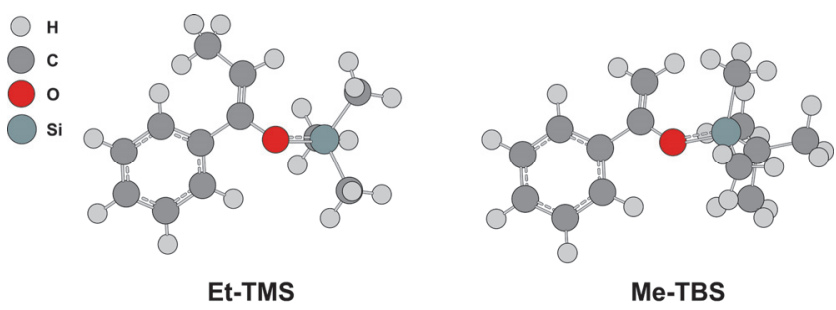

图 7 在 IEF-PCM M06-2X/6-31+G(d) 理论水平上优化得到的 Et-TMS 和 Me-TBS 结构

Figure 7 Optimized structures at IEF-PCM M06-2X/6-31+G(d) level of theory for Et-TMS and Me-TBS

图 8 是我们计算得到的 Et-TMS 与两性离子对中间 体 P- $\mathrm{H}_{2}$-split 发生 $\mathrm{H}^{+}$和 $\mathrm{H}^{-}$转移的吉布斯自由能面图. 在 $298.15 \mathrm{~K}, 101 \mathrm{kPa}$ 时, Et-TMS 与两性离子对中间体 P- $\mathrm{H}_{2}$-split形成复合物 EM-R-H ${ }^{+}$-transfer 是一个轻微吸热 的过程 $(12.3 \mathrm{~kJ} / \mathrm{mol})$, 该复合物需要经过过渡态 EM-TS-H ${ }^{+}$-transfer 并克服 $108.6 \mathrm{~kJ} / \mathrm{mol}$ 的能垒才能形成 EM-P- $\mathrm{H}^{+}$-transfer, $\mathrm{H}^{+}$转移是一个吸热过程 $(88.3 \mathrm{~kJ} / \mathrm{mol})$. 在 $\mathrm{EM}-\mathrm{R}-\mathrm{H}^{+}$-transfer 中, $\mathrm{P}-\mathrm{H}^{+}$键长为 $0.140 \mathrm{~nm}, \mathrm{H}^{+}$与 $\mathrm{C}^{-}$之间的距离为 $0.272 \mathrm{~nm}, \mathrm{C}=\mathrm{C}$ 键长为 $0.134 \mathrm{~nm}$; 过渡 态 EM-TS- $\mathrm{H}^{+}$-transfer 中 $\mathrm{P}-\mathrm{H}^{+}$键拉长至 $0.197 \mathrm{~nm}, \mathrm{H}^{+}$ 与 $\mathrm{C}^{-}$之间的距离缩短至 $0.128 \mathrm{~nm}, \mathrm{C}=\mathrm{C}$ 键长轻微拉长 至 $0.141 \mathrm{~nm}$, 表明: $\mathrm{H}^{+}$已向 $\mathrm{C}^{-}$转移, $\mathrm{C}=\mathrm{C}$ 已被部分活 化; 当生成 EM-P- $\mathrm{H}^{+}$-transfer 时, $\mathrm{H}^{+}$已完全离开 $\mathrm{P}$, 两者 之间的距离达 $0.288 \mathrm{~nm}, \mathrm{H}^{+}$与 $\mathrm{C}^{-}$形成共价键, 键长为 $0.110 \mathrm{~nm}, \mathrm{C}=\mathrm{C}$ 继续拉长至 $0.148 \mathrm{~nm}$, 已有部分单键的 特征. 该过程与 Me-TMS 中基本类似，但是其需要克服 的能垒更低, 吸热更少. 随后, EM-P- $\mathrm{H}^{+}$-transfer 也需要 经过重排及其随后的 $\mathrm{H}^{-}$转移使体系能量下降，但是令 人意外的是, 经过多次尝试我们并没有优化得到一个结 构类似于 MM-R- $\mathrm{H}^{-}$-transfer 有利于 $\mathrm{H}^{-}$转移的构型, 我 


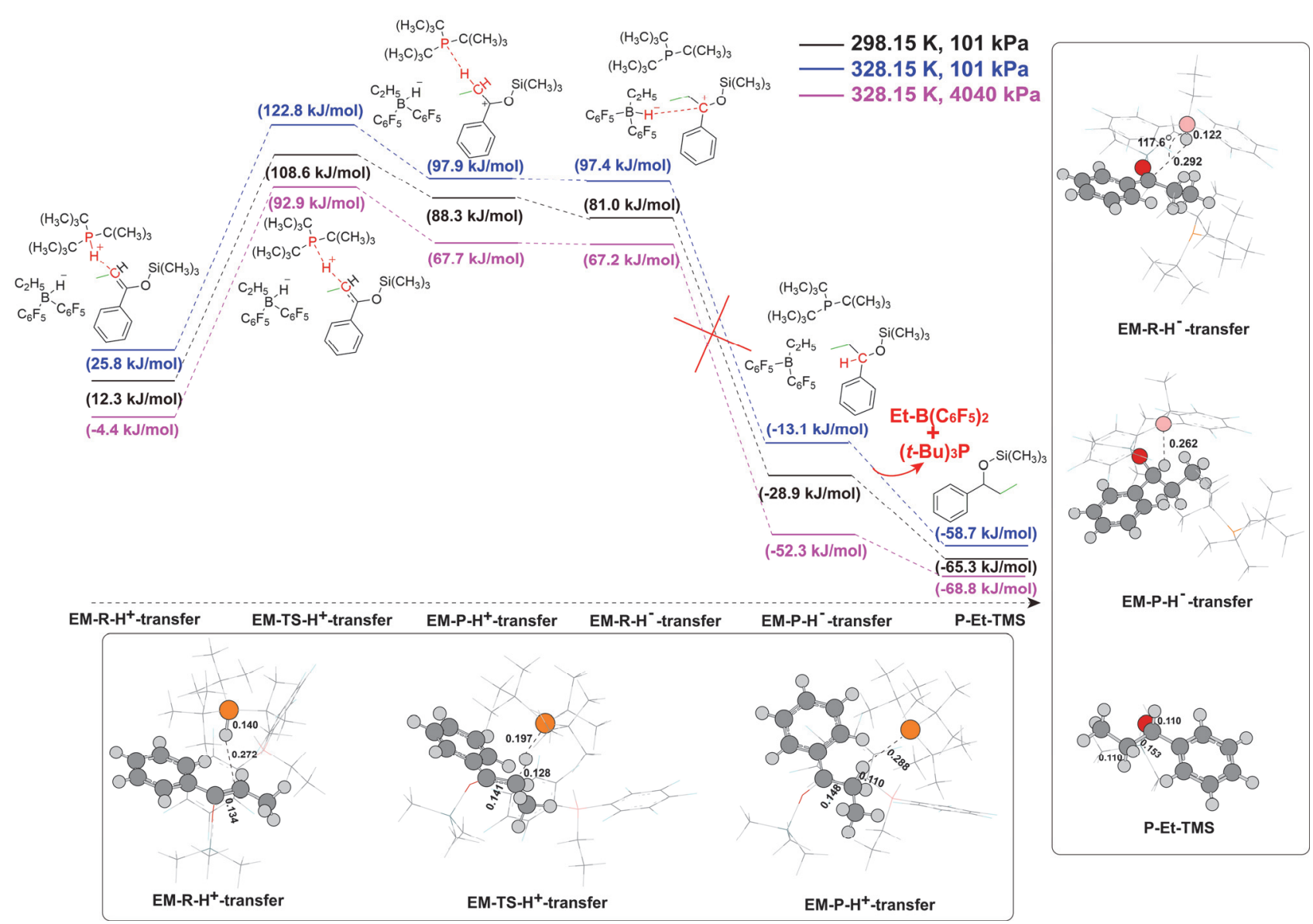

图 8 优化得到的两性离子与 Et-TMS 的 $\mathrm{H}^{+}$和 $\mathrm{H}^{-}$转移吉布斯自由能曲线, 其中优化的结构及其相对能量分别在 IEF-PCM M06-2X/6-31+G(d)和 IEF-PCM M06-2X/6-311+ $+\mathrm{G}(\mathrm{d}, \mathrm{p})$ 理论水平获得, 键长单位为 $\mathrm{nm}$

Figure 8 Optimized structures as well as Gibbs free energy profile of $\mathrm{H}^{+}$and $\mathrm{H}^{-}$transfer between intermediate of ion pair and Et-TMS. The structures and energies reported in this figure were calculated at the IEF-PCM M06-2X/6-31+G(d) and IEF-PCM M06-2X/6-311+ +G(d,p) level of theory, respectively. Selected bond distances are given in $\mathrm{nm}$

们优化得到的 EM-R-H - -transfer 构型如吉布斯自由能面 中所示. 在该结构中, $\mathrm{B}-\mathrm{H}^{-}$键长为 $0.122 \mathrm{~nm}$, 但是 $\mathrm{H}^{-}$ 与 $\mathrm{C}^{+}$的距离为 $0.292 \mathrm{~nm}$, 比 $\mathrm{MM}-\mathrm{R}-\mathrm{H}^{-}$-transfer $(0.224$ $\mathrm{nm}$ )更大, 也比文献报道的类似过程中 $\mathrm{H}^{-}$与 $\mathrm{C}^{+}$的距离 大 $(0.22 \sim 0.23 \mathrm{~nm}), \mathrm{B}-\mathrm{H}^{-} \cdots \mathrm{C}^{+}$之间的角度为 $117.6^{\circ}$, 该 构型明显不利于 $\mathrm{H}^{-}$向 $\mathrm{C}^{+}$的转移. 势能面扫描显示: 随 着 $\mathrm{H}^{-}$向 $\mathrm{C}^{+}$靠近能量持续上升(图 9). 这些结果意味着: $\mathrm{H}^{-}$可能不能向质子化的 Et-TMS 转移形成更加稳定的 EM-P-H ${ }^{-}$-transfer 及 P-Et-TMS.

在 $328.15 \mathrm{~K}, 101 \mathrm{kPa}$ 时, 吉布斯自由能曲线和 $298.15 \mathrm{~K}$ 时类似, 各中间体和过渡态以及产物的能量都 相对上升, $\mathrm{H}^{+}$转移的能垒上升至 $122.8 \mathrm{~kJ} / \mathrm{mol}$, 吸热稍 微上升至 $97.9 \mathrm{~kJ} / \mathrm{mol}$, 重排产物 EM-R- $\mathrm{H}^{-}$-transfer 的稳 定性与 EM-P-H $\mathrm{H}^{+}$-transfer 相当. 压力的升高也会使各中 间体、过渡态和产物的能量下降.

随后，我们使用相同的方法对 Me-TBS 与两性离子 对中间体 $\mathrm{P}-\mathrm{H}_{2}$-split 发生 $\mathrm{H}^{+}$和 $\mathrm{H}^{-}$转移的吉布斯自由能 曲线进行了计算. 其过程及自由能曲线中各物质的结构 与 Et-TMS 类似, 都是 Me-TBS 与 $\mathrm{P}-\mathrm{H}_{2}$-split 形成复合物 后经过过渡态 MB-TS- $\mathrm{H}^{+}$-transfer 发生 $\mathrm{H}^{+}$转移生成
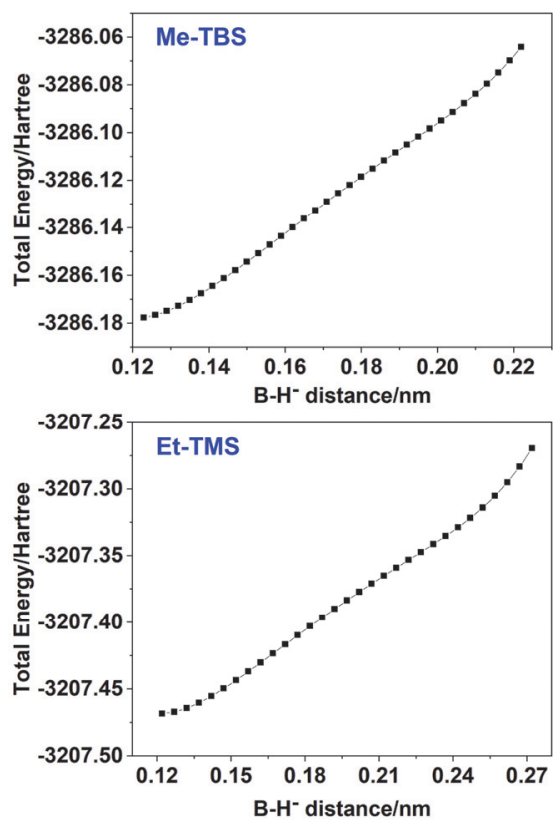

图 9 IEF-PCM M06-2X/6-31+G(d)理论水平扫描 Et-TMS 和 Me-TBS 中 $\mathrm{H}^{-}$转移势能面

Figure 9 Total energy scan of hydride transfer for Et-TMS and Me-TBS at IEF-PCM M06-2X/6-31+ G(d) level of theory 
MB-P-H ${ }^{+}$-transfer, 然后 MB-P-H ${ }^{+}$-transfer 再发生重排生 成利于 $\mathrm{H}^{-}$转移的构型(图 10). 但是从优化的构型看出: 在 MB-R-H $\mathrm{H}^{-}$-transfer 中, $\mathrm{H}^{-}$与 $\mathrm{C}^{+}$的距离为 $0.250 \mathrm{~nm}$, 稍 小于 $\mathrm{EM}-\mathrm{R}-\mathrm{H}^{-}$-transfer, $\mathrm{B}-\mathrm{H}^{-}$与 $\mathrm{C}^{+}$之间的角度为 $139.5^{\circ}$, 该角度虽然比 EM-R-H ${ }^{-}$-transfer 中更接近直线, 然而仍不利于 $\mathrm{H}^{-}$向 $\mathrm{C}^{+}$的转移. 势能面扫描结果显示: 随着 $\mathrm{H}^{-}$与 $\mathrm{C}^{+}$之间距离逐渐减小, 体系能量持续上升(图 9). 很明显, $\mathrm{H}^{-}$并不能转移至已被质子化的 Me-TBS 上 形成氢化产物, 与 Et-TMS 类似.

298.15 和 $328.15 \mathrm{~K}, 101 \mathrm{kPa}$ 时及 $328.15 \mathrm{~K}, 4040 \mathrm{kPa}$ 时计算的各物质的吉布斯自由能如图 10 所示. 在这些 条件下 MB-R-H ${ }^{+}$-transfer 的形成仍旧是一个去稳定化的 作用, 吸热分别为 $21.1,33.6$ 和 $3.6 \mathrm{~kJ} / \mathrm{mol} ; \mathrm{H}^{+}$转移需要 克服的能垒分别为 $107.2,119.2$ 和 $89.0 \mathrm{~kJ} / \mathrm{mol}$, 吸热分 别为 $81.0,88.9$ 和 $58.6 \mathrm{~kJ} / \mathrm{mol}$; 重排过程是一个放热过 程, 放热分别为 $12.5,5.5$ 和 $5.5 \mathrm{~kJ} / \mathrm{mol}$.

实际上，我们在前期的工作中，在相同的反应条件 下也进行了 Et-TMS 和 Me-TBS 的实验, 但是并没有得 到对应的氢化产物, 这进一步证实了我们关于 B-P-FLPs 催化的 Et-TMS 和 Me-TBS 氢化反应机理计算
的结果.

\subsection{B-P-FLPS 催化的烯醇硅醚氢化反应机理}

根据我们上文的计算结果, FLPs 催化的烯醇硅醚氢 化反应是从 $\mathrm{Et}-\mathrm{B}\left(\mathrm{C}_{6} \mathrm{~F}_{5}\right)_{2}$ 与 $t-\mathrm{Bu}_{3} \mathrm{P}$ 形成 FLPs 开始, 虽然 FLPs 的形成及后续与 $\mathrm{H}_{2}$ 作用形成 $\mathrm{R}-\mathrm{H}_{2}$-split 是一个持 续吸热的过程, 但是因为体系中存在有大量的 $\mathrm{H}_{2}$, 即使 形成的 R-H $\mathrm{H}_{2}$-split 通过去稳定作用返回到 FLPs, 其又会 与 $\mathrm{H}_{2}$ 作用生成 $\mathrm{R}-\mathrm{H}_{2}$-split, 大量 $\mathrm{H}_{2}$ 的存在是该反应能顺 利进行的前提. 当体系中生成 $\mathrm{R}-\mathrm{H}_{2}$-split 后, $\mathrm{H}_{2}$ 裂解过程 只需要克服 $74.0 \mathrm{~kJ} / \mathrm{mol}$ 的能量就能生成比 B-P-FLPs 更 稳定的中间产物 $\mathrm{P}-\mathrm{H}_{2}$-split $(-6.8 \mathrm{~kJ} / \mathrm{mol})$. 该物质是一 个两性离子对中间产物, 是 FLPs 催化的不饱和化合物 加氢反应中重要的活性中间产物. 反应底物 Me-TMS 的 加入也是一个吸热的过程, 但是考虑到体系中 Me-TMS 的量要远大于生成的 $\mathrm{P}-\mathrm{H}_{2}$-split, 所以认为该体系中是 存在有 $\mathrm{MM}-\mathrm{R}-\mathrm{H}^{+}$-transfer 的, 在高压条件下, $\mathrm{H}^{+}$转移从 动力学的角度是可行的, 但却是一个吸热的过程, 这似 乎是不利于氢化反应进行的，然而考虑到 $\mathrm{MM}-\mathrm{P}-\mathrm{H}^{+}$transfer 经重排及后续的 $\mathrm{H}^{-}$转移将持续放热生成更加

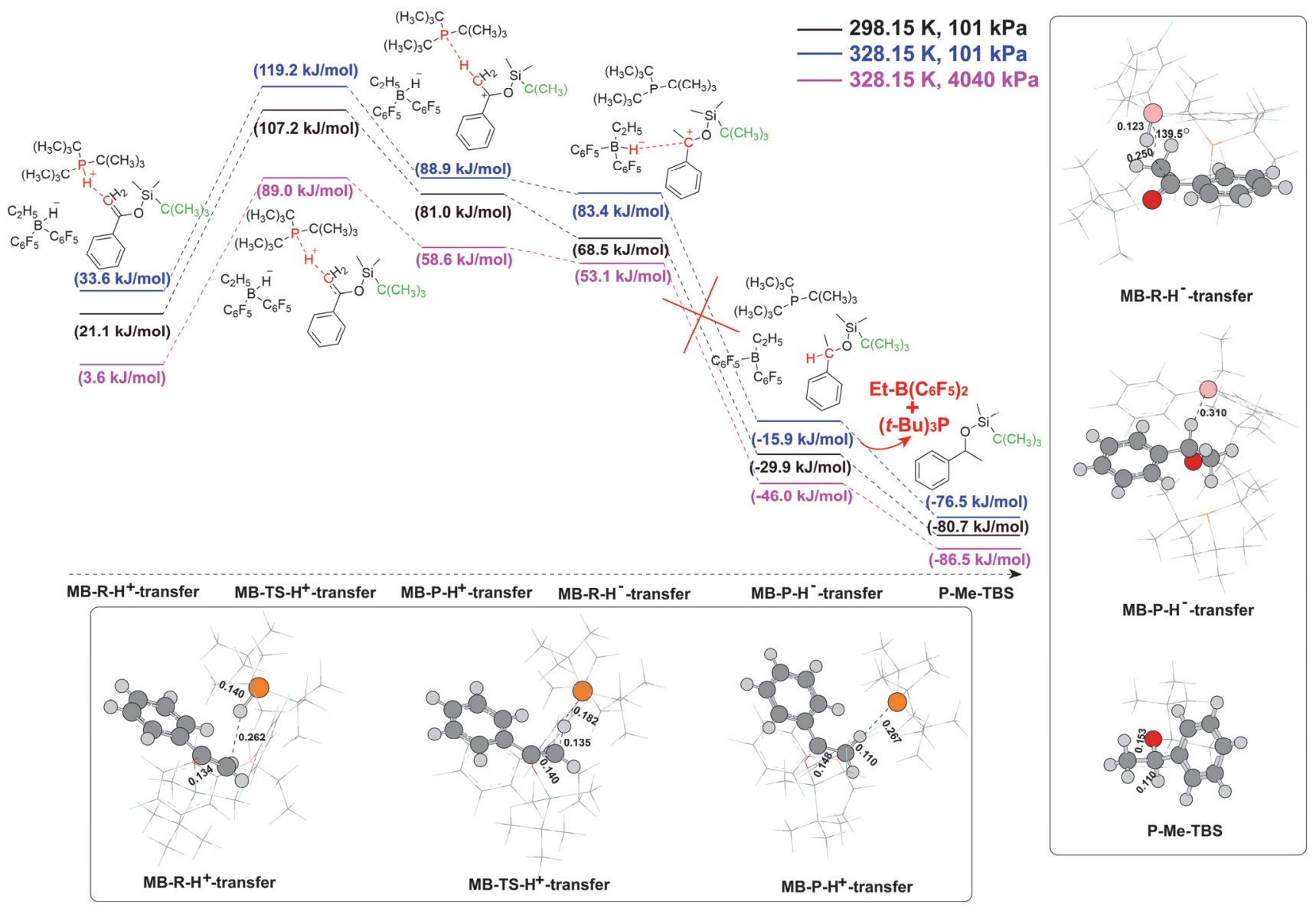

图 10 优化得到的两性离子与 Me-TBS 的 $\mathrm{H}^{+}$和 $\mathrm{H}^{-}$转移吉布斯自由能曲线, 其中优化的结构及其相对能量分别在 IEF-PCM M06-2X/6-31+ G(d) 和 IEF-PCM M06-2X/6-311+ $+\mathrm{G}(\mathrm{d}, \mathrm{p})$ 理论水平获得, 键长单位为 $\mathrm{nm}$

Figure 10 Optimized structures as well as Gibbs free energy profile of $\mathrm{H}^{+}$and $\mathrm{H}^{-}$transfer between intermediate of ion pair and Me-TBS. The structures and energies reported in this figure were calculated at the IEF-PCM M06-2X/6-31+G(d) and IEF-PCM M06-2X/6-311+ + G(d,p) level of theory, respectively. Selected bond distances are given in $\mathrm{nm}$ 
稳定的产物, 所以反应将向生成产物的方向持续进行.

在 FLPs 催化的 Et-TMS 和 Me-TBS 氢化反应中, 势 能面中各物质能量的变化趋势与 Me-TMS 中基本类似, 但是因为 $\mathrm{H}^{+}$转移后形成的产物并不能经过重排生成利 于 $\mathrm{H}^{-}$转移的中间体, 并且 $\mathrm{H}^{-}$转移过程需要持续吸热, 所以反应将朝着逆反应的方向进行, 直到生成一个相对 稳定的产物. 从我们计算的吉布斯自由能曲线中可以看 出, 在 FLPs 催化的 Et-TMS 和 Me-TBS 氢化反应中, 反 应可能停留在两性离子对中间体 $\mathrm{P}-\mathrm{H}_{2}$-split. 因为实验 体系中我们使用催化量的 $\mathrm{HB}\left(\mathrm{C}_{6} \mathrm{~F}_{5}\right)_{2}$ 和 $t-\mathrm{Bu}_{3} \mathrm{P}$, 所以体 系中生成的 $\mathrm{P}-\mathrm{H}_{2}$-split 也很少, 我们并未能观察到相应 物质的生成.

\section{3 结论}

本工作中, 我们以乙基取代的全氟苯基嗍作为路易 斯酸 $\left(\mathrm{Et}-\mathrm{B}\left(\mathrm{C}_{6} \mathrm{~F}_{5}\right)_{2}\right)$, 三叔丁基膦 $\left(t-\mathrm{Bu}_{3} \mathrm{P}\right)$ 作为路易斯碱, 烯醇硅醚化的苯乙酮(Me-TMS)作为底物建立了模型反 应, 然后使用密度泛函 M06-2X 并结合 6-31+G(d) 和 $6-311++\mathrm{G}(\mathrm{d}, \mathrm{p})$ 基组系统研究了 FLPs 催化的烯醇硅醚 氢化反应机理, 构型优化及单点能计算中的溶剂化效应 使用积分方程形式的极化连续介质模型(IEF-PCM)模 拟. 我们的计算结果发现: FLPs 催化的烯醇硅醚氢化反 应是从 $\mathrm{Et}-\mathrm{B}\left(\mathrm{C}_{6} \mathrm{~F}_{5}\right)_{2}$ 和 $t-\mathrm{Bu}_{3} \mathrm{P}$ 形成 B-P-FLPs 开始, 随后 会经过 $\mathrm{H}_{2}$ 裂解, $\mathrm{H}^{+}$和 $\mathrm{H}^{-}$转移等过程. 其中 $\mathrm{H}^{+}$转移是整 个氢化反应的决速步, 在 $298.15 \mathrm{~K}, 101 \mathrm{kPa}$ 时能垒为 $125.4 \mathrm{~kJ} / \mathrm{mol}$; B-P-FLPs 的形成及 $\mathrm{H}^{+}$转移是吸热过程, 所以保证体系中 $\mathrm{H}_{2}$ 和烯醇硅醚化的苯乙酮含量对整个 氢化反应及其重要; $\mathrm{H}_{2}$ 裂解过程不管从热力学还是动力 学角度都很容易发生; $\mathrm{H}^{-}$转移是一个无势垒过程, 在 $298.15 \mathrm{~K}, 101 \mathrm{kPa}$ 时放热达 $104.7 \mathrm{~kJ} / \mathrm{mol} .328 .15 \mathrm{~K}, 101$ $\mathrm{kPa}$ 时计算的势能面显示: 升高温度不利于氢化反应发 生, 吉布斯自由能曲线中各反应物、中间体、过渡态和 产物的能量都上升. 在该温度下将压力升高至 4040 $\mathrm{kPa}$, 吉布斯自由能面中各物质的能量大幅下降, 其中 $\mathrm{H}_{2}$ 裂解和 $\mathrm{H}^{+}$转移的能垒分别下降至 74.0 和 107.9 $\mathrm{kJ} / \mathrm{mol}$. 三甲基硅烷烯醇硅醚化的苯丙酮(Et-TMS)及叔 丁基二甲基硅烷烯醇硅醚化的苯乙酮(Me-TBS)氢化反 应机理计算结果显示: 延长烷基端的碳链和增大硅烷上 取代基的位阻虽然不会影响 $\mathrm{H}^{+}$转移过程, 但是会影响 $\mathrm{H}^{-}$转移, 使其不能形成利于 $\mathrm{H}^{-}$转移的结构, 且 $\mathrm{H}^{-}$向 $\mathrm{C}^{+}$ 转移过程能量持续上升, 最终导致氢化反应不能发生. 我们的这些研究结果有望为设计新的 FLPs 催化的烯醇 硅醚氢化反应提供理论支持.

\section{4 计算方法}

本工作中涉及的反应物、中间体、过渡态以及产物 的构型均在 M06-2X/6-31+G(d)理论水平上计算获得, 并且我们在相同的理论水平上也进行了振动频率分析,
计算中得到的过渡态有且仅有一个虚频，而反应物、中 间体以及产物没有虚频. 在此基础上，我们依据反应途 径内禀坐标理论(IRC)对优化得到的过渡态进行了极小 能量途径计算, 以正确关联反应物和产物. 为了获得更 加精确的能量，我们对计算得到的各化合物在 M06-2X/6-311 + $+\mathrm{G}(\mathrm{d}, \mathrm{p})$ 理论水平进行了单点能计算. 吉布斯自由能曲线中所示的 $101 \mathrm{kPa}$ 下, 298.15 和 328.15 $\mathrm{K}$ 以及 $328.15 \mathrm{~K}, 4040 \mathrm{kPa}$ 下的能量都经过零点能校正, 校正方法如下：单点能十不同温度或压力下的吉布斯自 由能热修正值(thermal correction to Gibbs free energy). 单点能和吉布斯自由能的热修正值如表 S1 所示. 结构 优化及单点能计算中甲苯的溶剂化效应使用积分方程 形式的极化连续介质模型(IEF-PCM)模拟. 所有计算都 通过 Gaussian 16 程序包进行 ${ }^{[52]}$.

\section{References}

[1] Liu, W. P.; Sahoo, B.; Junge, K.; Beller, M. Acc. Chem. Res. 2018, $51,1858$.

[2] Wang, Q. Y.; Santos, S.; Urbina-Blanco, C. A.; Hernandez, W. Y.; Imperor-Clerc, M.; Vovk, E. I.; Marinova, M.; Ersen, O.; Baaziz, W.; Safonova, O. V.; Khodakov, A. Y.; Saeys, M.; Ordomsky, V. V. Appl. Catal. B-Environ. 2021, 290, 120036.

[3] Chen, S. N.; Deng, J.; Ye, C.; Xu, C. C.; Huai, L. Y.; Ling, X.; Li, J.; Li, X. Y. Chem. Eng. J. 2021, 410, 128825.

[4] Wang, W. L.; Niu, J. F.; Yang, Z. F. J. Hazard. Mater. 2021, 411, 121912.

[5] Ye, R. P.; Lin, L.; Li, Q. H.; Zhou, Z. F.; Wang, T. T.; Russell, C. K.; Adidharma, H.; Xu, Z. H.; Yao, Y. G.; Fan, M. H. Catal. Sci. Technol. 2018, $8,3428$.

[6] Song, J. J.; Huang, Z. F.; Pan, L.; Li, K.; Zhang, X. W.; Wang, L.; Zou, J. J. Appl. Catal. B-Environ. 2018, 227, 386.

[7] Schreier, M. R.; Pfund, B.; Guo, X. W.; Wenger, O. S. Chem. Sci. 2020, $11,8582$.

[8] Lux, S.; Baldauf-Sommerbauer, G.; Siebenhofer, M. ChemSusChem 2018, 11,3357 .

[9] Meemken, F.; Baiker, A. Chem. Rev. 2017, 117, 11522.

[10] Hu, S. B.; Chen, M. W.; Zhai, X. Y.; Zhou, Y. G. Acta Chim. Sinica 2018, 76, 103 (in Chinese). (胡书博, 陈木旺, 翟小勇, 周永贵, 化 学学报, 2018, 76, 103.)

[11] Liu, X.; Han, Z. B.; Wang, Z.; Ding, K. L. Acta Chim. Sinica 2014, 72,849 (in Chinese). (刘旭, 韩召斌, 王正, 丁奎岭, 化学学报, 2014, 72, 849.)

[12] Liu, Y. B.; Du, H. F. Acta Chim. Sinica 2014, 72, 771 (in Chinese). (刘勇兵, 杜海峰, 化学学报, 2014, 72, 771.)

[13] Meemken, F.; Rodriguez-Garcia, L. J. Phys. Chem. Lett. 2018, 9, 996.

[14] Xie, J. H.; Zhou, Q. L. Acta Chim. Sinica 2012, 70, 1427 (in Chinese). (谢建华, 周其林, 化学学报, 2012, 70, 1427.)

[15] Zhang, Q.; Liu, A.; Yu, H. Z.; Fu, Y. Acta Chim. Sinica 2018, 76, 113 (in Chinese). (张琪, 刘奥, 于海珠，傅尧，化学学报, 2018, 76, 113.)

[16] Schauermann, S. J. Phys. Chem. Lett. 2018, 9, 5555.

[17] Bai, Y. P.; Cui, C. M. Acta Chim. Sinica 2020, 78, 763 (in Chinese). (白云平, 崔春明, 化学学报, 2020, 78, 763.)

[18] Welch, G. C.; Juan, R. R. S.; Masuda, J. D.; Stephan, D. W. Science 2006, 314, 1124.

[19] Stephan, D. W. Acc. Chem. Res. 2015, 48, 306

[20] Mömming, C. M.; Frömel, S.; Kehr, G.; Fröhlich, R.; Grimme, S.; Erker, G. J. Am. Chem. Soc. 2009, 131, 12280.

[21] Mahdi, T.; Stephan, D. W. J. Am. Chem. Soc. 2014, 136, 15809.

[22] Mahdi, T.; Heiden, Z. M.; Grimme, S.; Stephan, D. W. J. Am. Chem. Soc. 2012, 134, 4088.

[23] Zhang, Z.; Du, H. Angew. Chem. Int. Ed. 2015, 54, 623.

[24] Zhang, Z. H.; Du, H. F. Org. Lett. 2015, 17, 6266.

[25] Wei, S. M.; Feng, X. Q.; Du, H. F. Org. Biomol. Chem. 2016, 14, 8026.

[26] Wei, S. M.; Du, H. F. J. Am. Chem. Soc. 2014, 136, 12261. 
[27] Liu, Y. B.; Du, H. F. J. Am. Chem. Soc. 2013, 135, 6810.

[28] Liu, Y. B.; Du, H. F. J. Am. Chem. Soc. 2013, 135, 12968.

[29] Lu, Z. P.; Cheng, Z. H.; Chen, Z. X.; Weng, L. H.; Li, Z. H.; Wang, H. D. Angew Chem-Int. Ed. 2011, 50, 12227.

[30] Liu, Q.; Yang, L.; Yao, C.; Geng, J.; Wu, Y.; Hu, X. Org. Lett. 2021, 23,3685 .

[31] Rouf, A. M.; Huang, Y.; Dong, S.; Zhu, J. Inorg. Chem. 2021, 60, 5598.

[32] Wang, H. L.; Zhang, W. N.; Lu, L.; Liu, D. P.; Liu, D. D.; Li, T. Z.; Yan, S. C.; Zhao, S. Q.; Zou, Z. G. Appl. Catal. B-Environ. 2021, 283, 119639.

[33] Szynkiewicz, N.; Chojnacki, J.; Grubba, R. Inorg. Chem. 2020, 59, 6332.

[34] Adenot, A.; von Wolff, N.; Lefevre, G.; Berthet, J. C.; Thuery, P.; Cantat, T. Chem.-Eur. J. 2019, 25, 8118 .

[35] Kehr, G.; Erker, G. Chem. Rec. 2017, 17, 803.

[36] Wang, H. D.; Frohlich, R.; Kehr, G.; Erker, G. Chem. Commun. 2008, 5966.

[37] Greb, L.; Ona-Burgos, P.; Kubas, A.; Falk, F. C.; Breher, F.; Fink, K.; Paradies, J. Dalton Trans. 2012, 41, 9056.

[38] Ren, X. Y.; Du, H. F. J. Am. Chem. Soc. 2016, 138, 810

[39] Zhao, Y.; Truhlar, D. G. Theor. Chem. Acc. 2008, 120, 215

[40] Wang, Y. H.; Jie, J. L.; Zhao, H. M.; Bai, Y.; Qin, P. X.; Song, D. Acta Chim. Sinica 2018, 76, 475 (in Chinese). (王英辉, 节家龙, 赵 红梅, 白羽, 秦佩萱, 宋迪, 化学学报, 2018, 76, 475.)

[41] Wei, S.; Zhang, Z.; Liu, S.; Wang, Y. New J. Chem. 2021, 45, 11202.

[42] Huang, F.; Jiang, J. L.; Wen, M. W.; Wang, Z. X. J. Theor. Comput. Chem. 2014, 13, 1350074

[43] Wang, Y. H.; Wei, S. M.; Wang, K.; Xu, R. R.; Zhao, H. M. Acta Chim. Sinica 2020, 78, 271 (in Chinese). (王英辉, 魏思敏, 王康, 徐蓉蓉, 赵红梅, 化学学报, 2020, 78, 271.)

[44] Zhao, J. Y.; Wang, G. Q.; Li, S. H. Dalton Trans. 2015, 44, 9200

[45] Rokob, T. A.; Hamza, A.; Stirling, A.; Pápai, I. J. Am. Chem. Soc. 2009, 131, 2029
[46] Antinolo, A.; Carrillo-Hermosilla, F.; Fernandez-Galan, R.; Martinez-Ferrer, J.; Alonso-Moreno, C.; Bravo, I.; Moreno-Blazquez, S. Salgado, M.; Villasenor, E.; Albaladejo, J. Dalton Trans. 2016, 45 10717.

[47] Zhao, L.; Li, H.; Lu, G.; Huang, F.; Zhang, C.; Wang, Z.-X. Dalton Trans. 2011, 40, 1929.

[48] Rokob, T. A.; Hamza, A.; Papai, I. J. Am. Chem. Soc. 2009, 131, 10701.

[49] Wei, S. M.; Wang, Y. H.; Zhao, H. M. Acta Chim. Sinica 2019, 77, 278 (in Chinese). (魏思敏, 王英辉, 赵红梅, 化学学报, 2019, 77 278.)

[50] Cances, E.; Mennucci, B.; Tomasi, J. J. Chem. Phys. 1997, 107, 3032

[51] Das, S.; Pati, S. K. Chem.-Eur. J. 2017, 23, 1078.

[52] Frisch, M. J.; Trucks, G. W.; Schlegel, H. B.; Scuseria, G. E.; Robb, M. A.; Cheeseman, J. R.; Montgomery, J. A.; Vreven, T.; Kudin, K. N.; Burant, J. C.; Millam, J. M.; Iyengar, S. S.; Tomasi, J.; Barone, V.; Mennucci, B.; Cossi, M.; Scalmani, G.; Rega, N.; Petersson, G. A.; Nakatsuji, H.; Hada, M.; Ehara, M.; Toyota, K.; Fukuda, R.; Ha-segawa, J.; Ishida, M.; Nakajima, T.; Honda, Y.; Kitao, O.; Nakai, H.; Klene, M.; Li, X.; Knox, J. E.; Hratchian, H. P.; Cross, J. B.; Bakken, V.; Adamo, C.; Jaramillo, J.; Gomperts, R.; Stratmann, R. E.; Yazyev, O.; Austin, A. J.; Cammi, R.; Pomelli, C.; Ochterski, J. W.; Ayala, P. Y.; Morokuma, K.; Voth, G. A.; Salvador, P.; Dannenberg, J. J.; Zakrzewski, V. G.; Dapprich, S.; Daniels, A. D.; Strain, M. C.; Farkas, O.; Malick, D. K.; Rabuck, A. D.; Raghavachari, K.; Foresman, J. B.; Ortiz, J. V.; Cui, Q.; Baboul, A G.; Clifford, S.; Cioslowski, J.; Stefanov, B. B.; Liu, G.; Liashenko, A.; Piskorz, P.; Komaromi, I.; Martin, R. L.; Fox, D. J.; Keith, T.; Al-Laham, M. A.; Peng, C. Y.; Nanayakkara, A.; Challacombe, M.; Gill, P. M. W; Johnson, B; Chen, W; Wong, M. W; Gonzalez, C.; Pople, J. A. Gaussian 16, Revision A. 03, Gaussian, Inc., Wallingford, CT, 2016

(Cheng, B.) 